\title{
Influence of multi-walled carbon nanotubes on the thermoelectric properties of La-filled $\mathrm{CoSb}_{3}$ skutterudite composites
}

\author{
Ping Che ${ }^{1, *}$ Beibei Wang ${ }^{1}$, Changyan $\mathrm{Sun}^{1}$, Yongsheng $\mathrm{Han}^{2}$ and Wenjun $\mathrm{Li}^{1}$ \\ 1. Beijing Key Laboratory for Science and Application of Functional Molecular and Crystalline Materials, \\ Department of Chemistry, School of Chemistry and Biological Engineering, University of Science \& Technology \\ Beijing, Beijing 100083, China
}

2. State Key Laboratory of Multiphase Complex Systems, Institute of Process Engineering,

Chinese Academy of Sciences, Beijing 100190, China.

Key words: $\mathrm{CoSb}_{3}$ Carbon nanotubes Composites Thermoelectric properties

\begin{abstract}
Ideal thermoelectric materials are featured with high electrical and low thermal conduction. Oxide nanoparticles are usually added to these materials to increase their thermoelectric properties. The conductive particles are seldom employed since they have a complex effect on thermoelectric properties. Here we select the multi-walled carbon nanotubes (MWCNTs) as additives to synthesize a composite of La-filled $\mathrm{CoSb}_{3}$ skutterudite via a solvothermal method. The electrical conductivities and Seebeck coefficients of the obtained composites are tested by using a four-probe technique. The thermal conductivities are measured by a thermal diffusivity system using Pyroceram as a reference sample. The thermoelectric properties of the La-filled $\mathrm{CoSb}_{3} / \mathrm{MWCNTs}$ composites are investigated in the temperature range of 300-800 K. The influence of the MWCNTs on the electrical conductivity, the Seebeck coefficient

${ }^{*}$ Corresponding author.

E-mail address: cheping@ustb.edu.cn.
\end{abstract}


and the thermal conductivity of the La-filled $\mathrm{CoSb}_{3}$ /MWCNTs composites are discussed. The maximum figure of merit of the composites at $780 \mathrm{~K}$ is improved by $37 \%$.

Key words: Skutterudite; Thermoelectric properties; Multi-walled carbon nanotubes; Lanthanum filled

\section{Introduction}

Because cobalt skutterudite compounds exhibit phonon-glass and electron-crystal (PGEC) features, these compounds have been extensively studied as promising thermoelectric materials[1-3]. Skutterudite compounds are regarded as some of the most promising thermoelectric materials in the high temperature range because of their potentially high figures of merit, which is represented by $\mathrm{MX}_{3}(\mathrm{M}=\mathrm{Co}, \mathrm{Rh}, \mathrm{Ir}$; $\mathrm{X}=\mathrm{P}, \mathrm{As}, \mathrm{Sb}$ ) with a cubic structure and the space group $\mathrm{Im}_{3}$. Unlike the other types of binary skutterudites, $\mathrm{CoSb}_{3}$ has attracted extensive interest because of its large Seebeck coefficient, high electrical conductivity, and the abundant supply of its constituent elements, which are less volatile and expensive than those used for other skutterudite compounds. However, the thermal conductivity of $\mathrm{CoSb}_{3}$ is too high for it to function as an efficient thermoelectric material. One effective approach to reducing the thermal conductivity is to fill the open 'cage' with rare earth elements and/or other metallic atoms $[4,5]$. The filling atoms can adjust the doping concentration while simultaneously maintaining a high Seebeck coefficient and improving the electrical 
conductivity. Filling atoms can also enhance the phonon scattering of the lattice, resulting in the reduction of thermal conductivity. Introducing nano-structures into the bulk phase matrix is another approach for improving the thermoelectric properties that have received attention over the last years[6]. Nanoparticles have the potential to decrease the thermal conduction and also to enhance the electrical properties. The former property is reduced, owing to the numerous nanoscale interfaces, thus leading to a limitation of the phonon mean free path. The latter feature is related to the quantum confinement effects and results in superior thermopower values. For example, Battabyal et al. have synthesized $\mathrm{Sn}_{0.4} \mathrm{Ba}_{0.4} \mathrm{Co}_{4} \mathrm{Sb}_{12}$ skutterudites dispersed with $\mathrm{Cu}_{2} \mathrm{O}$ nanoparticles and have found a maximum figure of merit $(\mathrm{ZT}) \sim 0.92$ achieved at $573 \mathrm{~K}$, which is more than $200 \%$ that of the pure $\mathrm{Sn}_{0.4} \mathrm{Ba}_{0.4} \mathrm{Co}_{4} \mathrm{Sb}_{12}$ sample[7].

The particles added to skutterudites are mainly oxide insulates in previous studies[8-10]. There are a few reports on the addition of conductive particles to this material since it was supposed that the conductive particles would increase both electrical and thermal conduction, resulting in a decrease of ZT value of the composites. However, this result is based on the assumption that the thermal increase wins the electrical increase, which is not always the truth. It therefore, deserves to make an investigation on this unclear issue. In this work, MWCNTs is selected as the conductive particles which are added to n-type $\mathrm{La}_{0.3} \mathrm{Co}_{4} \mathrm{Sb}_{12}$ thermoelectric materials via the solvothermal method. The thermoelectric properties of the skutterudite compounds were measured and the effect of MWCNTs amount on the thermoelectric 
properties were discussed in detail.

\section{Experimental}

The preparation of the samples containing La-filled $\mathrm{CoSb}_{3}$ and $\mathrm{x}$ wt.\% MWCNTs $(\mathrm{x}=0,1,2,4,6)$ composites in this study required several steps. First, MWCNTs purchased from Alfa Aesar was preprocessed by boiling in concentrated nitric acid to purify the carbon nanotubes and introduce organic functional groups to enhance the dispersion of the carbon nanotubes in ethanol. $\mathrm{La}_{0.3} \mathrm{CoSb}_{3}$ powders were prepared through the solvothermal method[11]. $\mathrm{La}_{2} \mathrm{O}_{3}, \mathrm{CoCl}_{2}, \mathrm{SbCl}_{3}$ and MWCNTs were well blended in ethanol with a certain amount of PVP as the surfactant, $\mathrm{NaBH}_{4}$ was added under ultrasonic dispersion, and the mixture was transferred into a quartz-lined autoclave filled to $85 \%$ of its volume. The autoclave was heated to $240^{\circ} \mathrm{C}$ for 48 hours and then cooled to room temperature; the solid products in the autoclave were filtered, washed with distilled water and ethanol several times, and then dried at $80^{\circ} \mathrm{C}$ for $5 \mathrm{~h}$ for characterization.

The obtained powders were analyzed through X-ray diffraction (XRD) with a RigakuD/MAX-2550PC diffractometer using $\mathrm{Cu} \mathrm{K \alpha}$ radiation $(\lambda=0.15406 \mathrm{~nm})$ in the range of $2 \theta=20-80^{\circ}$. The morphology of the obtained materials was observed with a JED-2300 JEOL with a Phoenix X-ray EDS.

Compaction of the samples was performed by spark plasma sintering (SPS) at 853 $\mathrm{K}$ for $10 \mathrm{~min}$ under the pressure of $40 \mathrm{MPa}$, thus resulting in pellets with a diameter of $15 \mathrm{~mm}$ or $10 \mathrm{~mm}$. The larger pellet was polished and cut into sticks with dimensions 
of $12 \mathrm{~mm} \times 4 \mathrm{~mm} \times 2 \mathrm{~mm}$ for measuring the electrical conductivity and the Seebeck coefficient, whereas the smaller pellet with a diameter of $10 \mathrm{~mm}$, which was prepared by hot pressing, was used for measuring the thermal conductivity.

\section{Results and discussion}

\subsection{Phase and microstructures}

Fig. 1 shows the typical XRD patterns of the $\mathrm{CoSb}_{3}$ and $\mathrm{La}$-filled $\mathrm{CoSb}_{3}$ powders prepared by solvothermal synthesis. As shown in the figure, the majority of the diffraction peaks could be indexed to the cubic skutterudite $\mathrm{CoSb}_{3}$ phase with the $\operatorname{Im} 3$ space group, and a small amount of $\mathrm{CoSb}_{2}$ mesophase was also be observed. The XRD peaks of the La-filled $\mathrm{CoSb}_{3}$ shifted slightly to lower angles because the La atom filled into the lattice cage of $\mathrm{CoSb}_{3}$, slightly increases the skutterudite unit cell volume; this slight increase was further proven by examination of the $\mathrm{CoSb}_{3}$ and $\mathrm{La}_{0.3} \mathrm{CoSb}_{3}$ lattice parameters. According to the XRD data of the sample, we calculated the lattice constants $\left(a\right.$ ) of the $\mathrm{CoSb}_{3}$ and La_filled $\mathrm{CoSb}_{3}$ to be $9.036 \AA$ and $9.040 \AA$, respectively. These values are similar to the standard lattice parameter of the pure $\mathrm{CoSb}_{3}$ phase[12] $(9.035 \AA)$ and the lattice parameter of the Yb-filled $\mathrm{CoSb}_{3}$ phase[13] (9.046 ̊), indicating that the calculated results are reliable.

Fig. 2 (a) shows the SEM images of the La-filled $\mathrm{CoSb}_{3}$ synthesized through the solvothermal method. Homogeneous granules with an average particle size of $\sim 300$ nm were observed. Fig. 2(b) shows the EDS spectrum of the selected region. The Co, $\mathrm{Sb}$ and La peaks are visible in Fig. 2(c) with ratios of 0.40: 5.44: 16.36, representing a 
slight shift from the $\mathrm{La}_{0.3} \mathrm{CoSb}_{3}$ stoichiometry. The $\mathrm{C}$ peak arose from the spraying of carbon during the SEM sample preparation, and the O peak may have been caused by adsorption of the sample. Compared to the sample prepared through solid-state reaction methods[14], the obtained materials showed greater uniformity and smaller particle sizes.

Fig. 3a shows the XRD patterns of the samples containing $\mathrm{La}_{0.3} \mathrm{CoSb}_{3}$ and $\mathrm{x}$ wt.\% MWCNTs. The major products were the skutterudite phase together with a small amount of the $\mathrm{CoSb}_{2}$ impurity. Owing to the small amount of the added MWCNTs, there were no obvious MWCNTs peaks. As shown in Fig. 3b, the MWCNTs were randomly dispersed in the skutterudite $\mathrm{La}_{0.3} \mathrm{CoSb}_{3}$ substrate and showed mutual crosslinking, forming a disorganized MWCNTs network when $\mathrm{x}$ was greater than $4 \%$, which may have resulted in a strong effect on the thermoelectric properties of the composite material. After SPS compaction, the size of the $\mathrm{La}_{0.3} \mathrm{CoSb}_{3}$ particles does not change obviously. While the grain boundaries are largely observed between particles, which scatters the phonons.

Fig. 4a and $\mathrm{b}$ show the morphologies of MWCNTs before and after purification. They have similar morphology, but have obvious difference for FT-IR spectra, as shown in Fig.4c. After purification, three peaks at $800.63 \mathrm{~cm}^{-1} 、 1025.41 \mathrm{~cm}^{-1}$ and 1250.10 $\mathrm{cm}^{-1}$ are observed. These peaks belong to the stretching vibration of $\mathrm{C}-\mathrm{O}$ and $\mathrm{C}=\mathrm{O}$ respectively, indicating the existence of carboxyl, hydroxyl and carbonyl group, which is caused by purification. 


\subsection{Thermoelectric properties}

The temperature dependence of the electrical conductivity is shown in Fig. 5. The conductivity $\sigma$ of $\mathrm{La}_{0.3} \mathrm{CoSb}_{3}+\mathrm{x}$ wt.\% MWCNTs composites increased with the temperature, a result consistent with the features of a semiconductor. At the same temperature, the $\sigma$ values of the composites increased with increasing amounts of MWCNTs. The results showed that the conductivity $\sigma$ of the mixture was improved with increasing MWCNTs content, mainly for the following reasons. 1) The La atom filling of the skutterudite $\mathrm{CoSb}_{3}$ formed a p-type semiconductor as observed from the analysis of the Seebeck coefficients described below. Therefore, although the conductivity $\sigma$ of $\mathrm{La}_{0.3} \mathrm{CoSb}_{3}$ also depends on the free electron concentration, the conductivity of the MWCNTs is higher. The conductivity $\sigma$ of MWCNTs reached $10^{6}-10^{8} \mathrm{~S} \cdot \mathrm{m}^{-1}$ at room temperature after functionalization and increases with the temperature, whereas that of the skutterudite $\mathrm{CoSb}_{3}$ was $\sim 10^{4} \mathrm{~S} \cdot \mathrm{m}^{-1} .2$ ) The second phase MWCNTs were distributed in the skutterudite $\mathrm{La}_{0.3} \mathrm{CoSb}_{3}$ and formed a distinct network structure, providing good pathways for electron transport in the samples with a high amount of MWCNTs. The carrier concentration of skutterudite $\mathrm{La}_{0.3} \mathrm{CoSb}_{3}$ was increased by MWCNTs doping and resulted in the improvement of conductivity $\sigma$.

Fig. 6 shows the Seebeck coefficients of the SPS-processed $\mathrm{La}_{0.3} \mathrm{CoSb}_{3}+\mathrm{x}$ wt. $\%$ MWCNTs bulk sample for several values of $\mathrm{x}$. The Seebeck coefficients of all the composites were negative, showing the characteristics of an n-type semiconductor. The results demonstrated that with increasing temperatures, the Seebeck coefficients of the composites first increased and then decreased, achieving maximum values 
between $500 \mathrm{~K}$ to $600 \mathrm{~K}$ depending on the $\mathrm{x}$ values. After $600 \mathrm{~K}$, the Seebeck coefficients began to decrease, mainly because of the high carrier concentration caused by the intrinsic excitation. At the same temperature, the results showed that increasing content of MWCNTs decreases the Seebeck coefficient of the composite. According to the semiconductor theory, the absolute value of the Seebeck coefficient is directly proportional to the effective mass of the carrier and is inversely proportional to the carrier concentration. As more MWCNTs are added, more electrons are provided to the composite material, resulting in the increased carrier concentration of the material and causing the absolute value of the Seebeck coefficient to decrease.

Fig. 7 shows the thermal conductivity of the $\mathrm{La}_{0.3} \mathrm{CoSb}_{3}+\mathrm{x}$ wt.\% (MWCNTs) processed by SPS compaction for several temperatures. The thermal conductivity $\kappa$ of the composite decreased with increasing temperature and then tended toward a stable value when the temperature was higher than $650 \mathrm{~K}$. At the same temperature, the thermal conductivity $\kappa$ of the composite increased with increasing MWCNTs content. However, as shown in the figure, there were no obvious changes in the thermal conductivities of composites with MWCNTs contents under 2 wt.\%. However, the thermal conductivity rose significantly for MWCNTs content higher than $4 \%$.

With the increase of the MWCNTs content, the thermal conductivity of the composite was improved mostly because MWCNTs itself possess a high thermal conductivity[15]. The thermal conductivity reached $10^{3} \mathrm{~W} \cdot \mathrm{m}^{-1} \cdot \mathrm{K}^{-1}$ at $\mathrm{room}$ temperature for MWCNTs either before[16] or after functionalization[17], whereas 
that of the skutterudite $\mathrm{CoSb}_{3}$ was $8 \mathrm{~W} \cdot \mathrm{m}^{-1} \cdot \mathrm{K}^{-1}$.

However, why does the thermal conductivity change slightly when the MWCNTs doping amount is less than $2 \%$ while showing an obvious rise when the additive amount is more than $4 \%$ ? First, MWCNTs distributed in the skutterudite $\mathrm{CoSb}_{3}$ at the second phase introduce large numbers of grain boundaries, dislocations and interfacial defects. The defects, including grain boundaries and dislocations, form many new lattice scattering centers, producing strong scattering of the movement of the phonon, and in theory, causing a decline in the thermal conductivity. Therefore, for the MWCNTs amount of $1-2 \%$, on the one hand, MWCNTs possess a high thermal conductivity, whereas, on the other hand, at the second phase, MWCNTs exhibit a clear phonon scattering effect; owing to the combination of the two factors, thermal conductivity changes slightly. Increasing the doping amount to 4- 6\% may cause more phonon scattering, but the effect of the high intrinsic thermal conductivity of MWCNTs dominates, resulting in the significant increase of the composite thermal conductivity.

Fig. 8 shows the dependence of the $\mathrm{ZT}$ values of the $\mathrm{La}_{0.3} \mathrm{CoSb}_{3}+\mathrm{x}$ wt.\% MWCNTs on the temperature. ZT values increased with increasing temperatures and tended to plateau near $800 \mathrm{~K}$. The ZT values at the same temperature first increased and then decreased with increasing amounts of MWCNTs and the highest values were obtained when the MWCNTs content was 2\%. Although the Seebeck coefficients showed a decreasing trend with increasing MWCNTs amounts, the thermal conductivity increased and the electrical conductivity of the composites dramatically 
improved; because $\mathrm{ZT}=\alpha^{2} \sigma \mathrm{T} / \kappa$, for the MWCNTs amount of $2 \%$, we obtained thermoelectric materials with $\mathrm{ZT}=0.26$ at $780 \mathrm{~K}$, which represents an enhancement of $37 \%$ compared with the $\mathrm{ZT}$ value of $\mathrm{La}_{0.3} \mathrm{CoSb}_{3}$.

\section{Conclusions}

In conclusion, composites with the La-filled $\mathrm{CoSb}_{3}$ and MWCNTs were synthesized through a solvothermal route. The thermoelectric properties of these materials were measured. Increasing the MWCNTs amount results in increases in the electrical conductivity and thermal conductivity of the composite, whereas the Seebeck coefficient decreases. However, the figure of merit (ZT) of the composites were dependent on the amount of the carbon nanotubes. With the amount of less than $2 \%$, the ZT values increase with the amounts, reaching a maximum at $2 \%$, which results in a $37 \%$ improvement over $\mathrm{La}_{0.3} \mathrm{CoSb}_{3}$ at $780 \mathrm{~K}$. The mechanism behind this improvement was discussed in this paper. The findings in this paper indicated that the addition of conductive particles to thermoelectric materials is a suitable solution to have an improved thermoelectric properties of materials.

\section{Acknowledgments}

This work was supported by the National Science Foundation of China (21290174) and the Fundamental Research Funds for the Central Universities (YJ2012-007). The authors are also grateful to State Key Laboratory of Rare Earth Resource Utilization for physical properties testing. 


\section{References}

[1] W.Y. Zhao, P. Wei, Q.J. Zhang, H. Peng, W.T. Zhu, D.G. Tang, J. Yu, H.Y. Zhou, Z.Y. Liu, X. Mu, D.Q. He, J.C. Li, C.L. Wang, X.F. Tang, J.H. Yang, Multi-localization transport behaviour in bulk thermoelectric materials, Nature communications, 6 (2015) 7.

[2] M. Gu, X.G. Xia, X.Y. Huang, S.Q. Bai, X.Y. Li, L.D. Chen, Study on the interfacial stability of p-type $\mathrm{Ti} / \mathrm{Ce}_{\mathrm{y}} \mathrm{Fe}_{\mathrm{x}} \mathrm{Co}_{4-\mathrm{x}} \mathrm{Sb}_{12}$ thermoelectric joints at high temperature, J. Alloys Compd., 671 (2016) 238-244.

[3] A. Gharleghi, Y.-H. Chu, F.-H. Lin, Z.-R. Yang, Y.-H. Pai, C.-J. Liu, Optimization and Analysis of Thermoelectric Properties of Unfilled $\mathrm{Co}_{1-\mathrm{x}-\mathrm{y}} \mathrm{Ni}_{\mathrm{x}} \mathrm{Fe}_{\mathrm{y}} \mathrm{Sb}_{3}$ Synthesized via a Rapid Hydrothermal Procedure, ACS Applied Materials \& Interfaces, 8 (2016) 5205-5215.

[4] L.L. Xi, Y.T. Qiu, X. Shi, W.Q. Zhang, L.D. Chen, D.J. Singh, J.H. Yang, Defect-enhanced void filling and novel filled phases of open-structure skutterudites, Chemical Communications, 51 (2015) 10823-10826.

[5] L.L. Xi, Y.T. Qiu, S. Zheng, X. Shi, J. Yang, L.D. Chen, D.J. Singh, J.H. Yang, W.Q. Zhang, Complex doping of group 13 elements In and $\mathrm{Ga}$ in caged skutterudite $\mathrm{CoSb}_{3}$, Acta Materialia, 85 (2015) 112-121.

[6] Z.M. He, C. Stiewe, D. Platzek, G. Karpinski, E. Mueller, S.H. Li, M. Toprak, M. Muhammed, Nano $\mathrm{ZrO}_{2} / \mathrm{CoSb}_{3}$ composites with improved thermoelectric figure of merit, Nanotechnology, 18 (2007) 5.

[7] M. Battabyal, B. Priyadarshini, D. Sivaprahasam, N.S. Karthiselva, R. Gopalan, The effect of $\mathrm{Cu}_{2} \mathrm{O}$ nanoparticle dispersion on the thermoelectric properties of n-type skutterudites, Journal of Physics D: Applied Physics, 48 (2015) 455309.

[8] C. Chubilleau, B. Lenoir, P. Masschelein, A. Dauscher, C. Candolfi, E. Guilmeau, C. Godart, Influence of $\mathrm{ZnO}$ nano-inclusions on the transport properties of the $\mathrm{CoSb}_{3}$ skutterudite, J. Alloys Compd., 554 (2013) 340-347.

[9] D.G. Zhao, M. Zuo, J.F. Leng, H.R. Geng, Synthesis and thermoelectric properties of $\mathrm{CoSb}_{3} / \mathrm{WO}_{3}$ thermoelectric composites, Intermetallics, 40 (2013) 71-75.

[10] G.J. Tan, Y. Zheng, X.F. Tang, High thermoelectric performance of nonequilibrium synthesized $\mathrm{CeFe}_{4} \mathrm{Sb}_{12}$ composite with multi-scaled nanostructures, Applied Physics Letters, 103 (2013) 5.

[11] J.L. Mi, X.B. Zhao, T.J. Zhu, J.P. Tu, Nanosized La filled $\mathrm{CoSb}(3)$ prepared by a solvothermal-annealing method, Materials Letters, 62 (2008) 2363-2365.

[12] N.R. Dilley, E.D. Bauer, M.B. Maple, B.C. Sales, Thermoelectric properties of chemically substituted skutterudites $\mathrm{Yb}_{\mathrm{y}} \mathrm{Co}_{4} \mathrm{Sn}_{\mathrm{x}} \mathrm{Sb}_{12-\mathrm{x}}$, Journal of Applied Physics, 88 (2000) 1948-1951.

[13] B. Alinejad, A. Castellero, M. Baricco, Full dense $\mathrm{CoSb}_{3}$ single phase with high thermoelectric performance prepared by oscillated cooling method, Scripta Materialia, 113 (2016) 110-113.

[14] E. Visnow, C.P. Heinrich, A. Schmitz, J. de Boor, P. Leidich, B. Klobes, R.P. Hermann, W.E. Muller, W. Tremel, On the True Indium Content of In-Filled Skutterudites, Inorganic Chemistry, 54 (2015) 7818-7827.

[15] W. Yi, L. Lu, Dian-Li Zhang, Z. W. Pan, S. S. Xie, Linear specific heat of carbon nanotubes, Phys. Rev. B, 59 (1999) 4.

[16] G.J. Hu, B.Y. Cao, Thermal conductivity of multi-walled carbon nanotubes: Molecular dynamics simulations, Chinese Physics B, 23 (2014) 7. 
[17] S. Sarbolookzadeh Harandi, A. Karimipour, M. Afrand, M. Akbari, A. D'Orazio, An experimental study on thermal conductivity of F-MWCNTs $-\mathrm{Fe}_{3} \mathrm{O}_{4} / \mathrm{EG}$ hybrid nanofluid: Effects of temperature and concentration, International Communications in Heat and Mass Transfer, 76 (2016) 171-177. 


\section{Captions}

Fig. 1. XRD patterns of $\mathrm{CoSb}_{3}$ and La-filled $\mathrm{CoSb}_{3}$

Fig. 2. SEM images of the La-filled $\mathrm{CoSb}_{3}$ samples (a) and the EDS (C) from the selected region (b).

Fig. 3. XRD patterns(a) of the samples containing $\mathrm{La}_{0.3} \mathrm{CoSb}_{3}$ and various weight percentage of MWNTS, and SEM image (b) of the sample containing $\mathrm{La}_{0.3} \mathrm{CoSb}_{3}$ and 4 wt.\% MWCNTs after SPS compaction

Fig. 4. Morphologies (a, b) and FT-IR spectra (C) of MWCNTs before and after purification

Fig. 5. Electrical conductivities of the samples containing $\mathrm{La}_{0.3} \mathrm{CoSb}_{3}$ and various weight percentage of MWCNTs compacted with SPS

Fig. 6. Seebeck coefficients of the samples containing $\mathrm{La}_{0.3} \mathrm{CoSb}_{3}$ and various weight percentage of MWCNTs compacted with SPS

Fig. 7. Thermal conductivity $\mathrm{k}$ of the samples containing $\mathrm{La}_{0.3} \mathrm{CoSb}_{3}$ and various weight percentage of MWCNTs compacted with SPS

Fig. 8. ZT values of the samples containing $\mathrm{La}_{0.3} \mathrm{CoSb}_{3}$ and various weight percentage of MWCNTs compacted with SPS 
Fig. 1

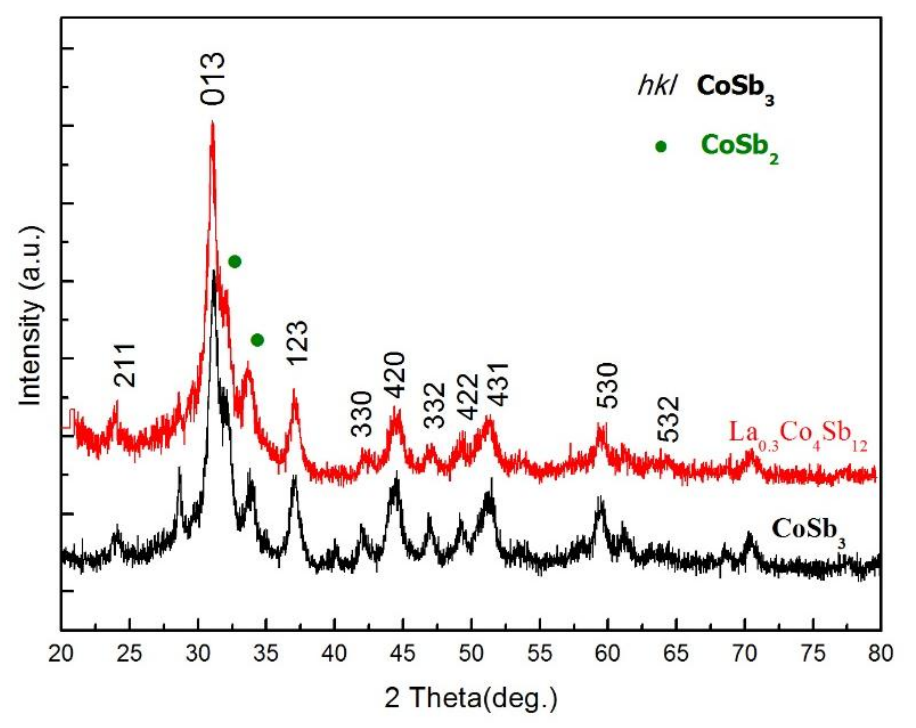

Fig. 1. XRD patterns of $\mathrm{CoSb}_{3}$ and La-filled $\mathrm{CoSb}_{3}$ 
Fig. 2
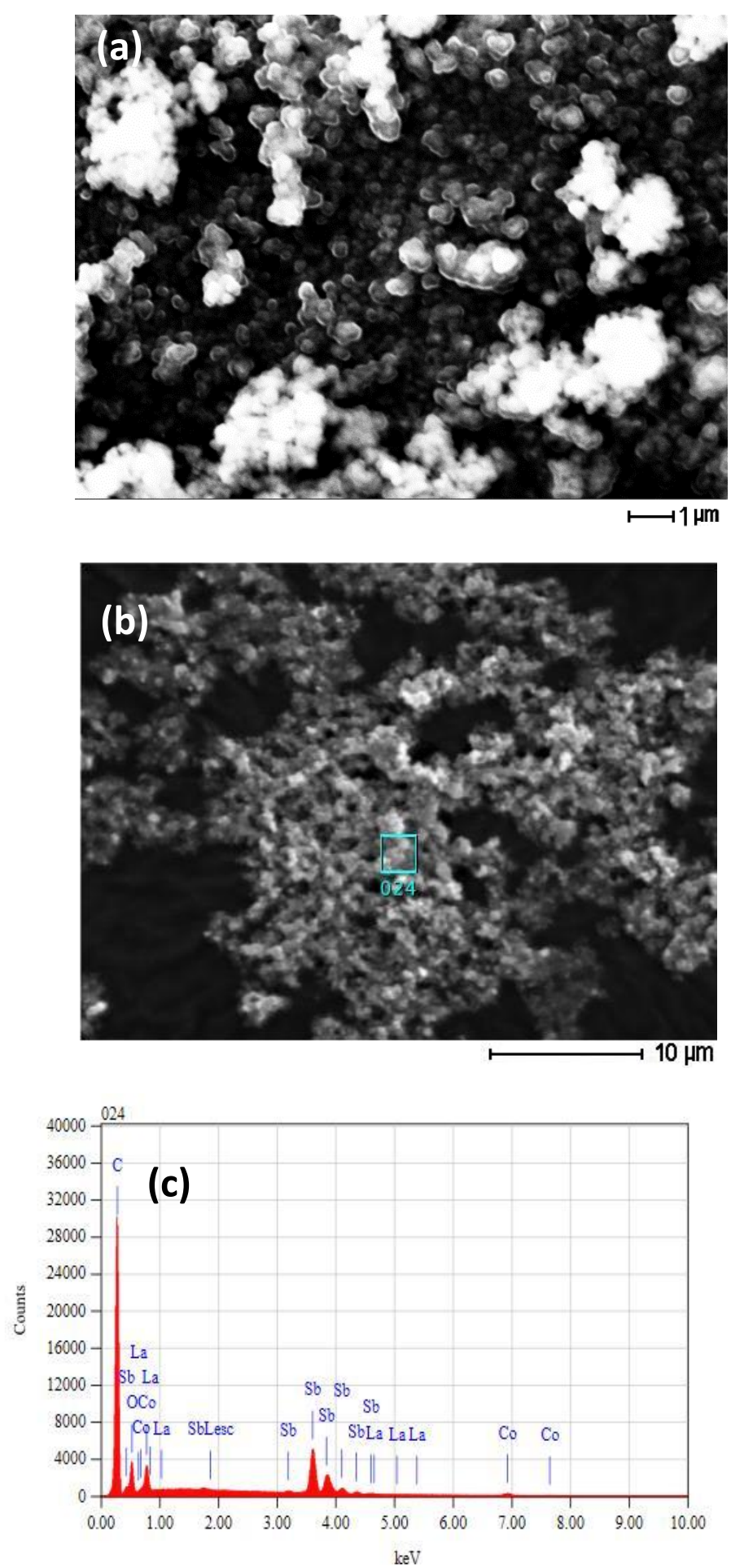

Fig. 2. SEM images of the La-filled $\operatorname{CoSb}_{3}$ samples (a) and the EDS (C) from the selected region (b). 
Fig. 3
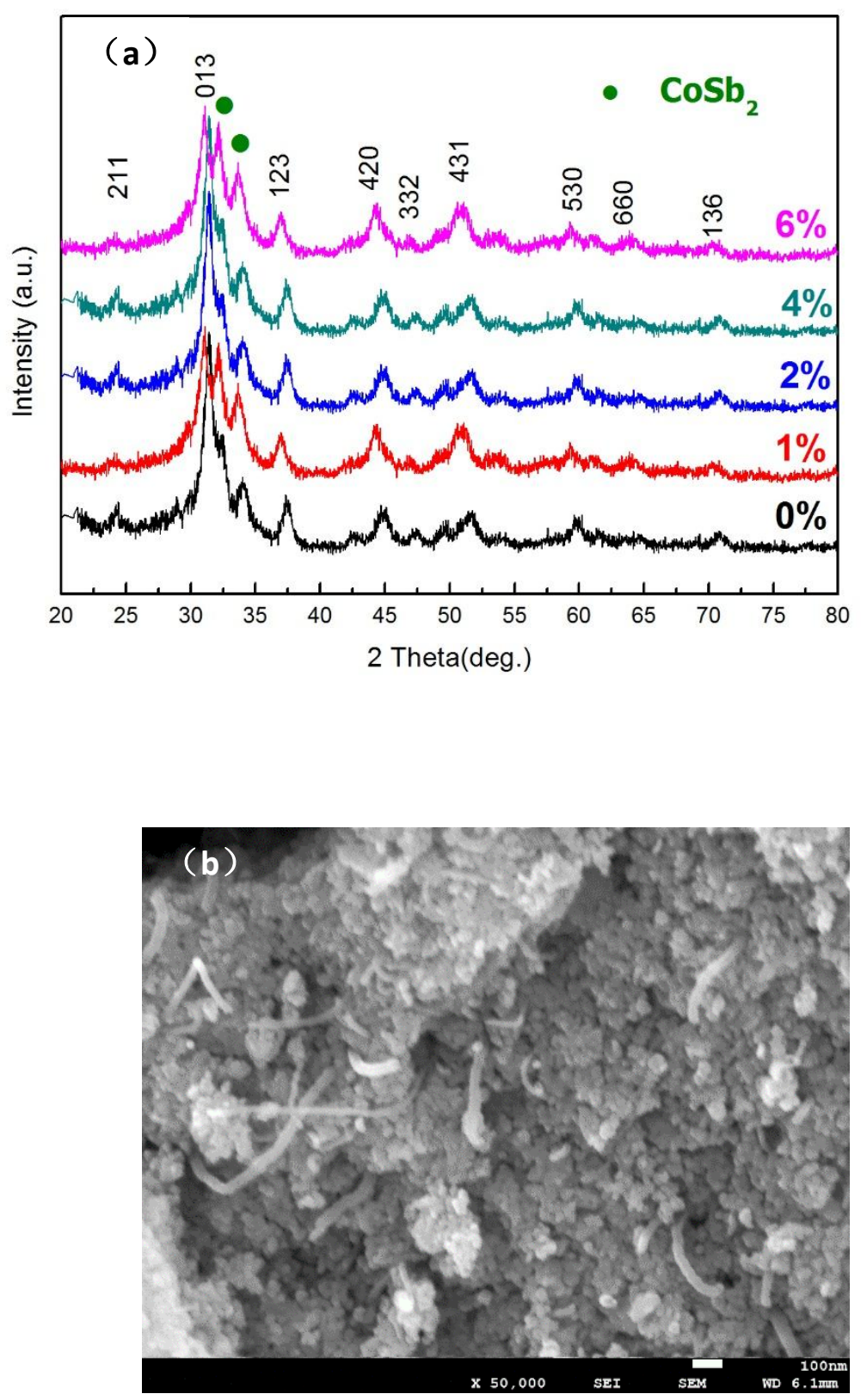

Fig. 3. XRD patterns(a) of the samples containing $\mathrm{La}_{0.3} \mathrm{CoSb}_{3}$ and various weight percentage of MWNTS, and SEM image (b) of the sample containing $\mathrm{La}_{0.3} \mathrm{CoSb}_{3}$ and 4 wt. \% MWCNTs after SPS compaction 
Fig. 4
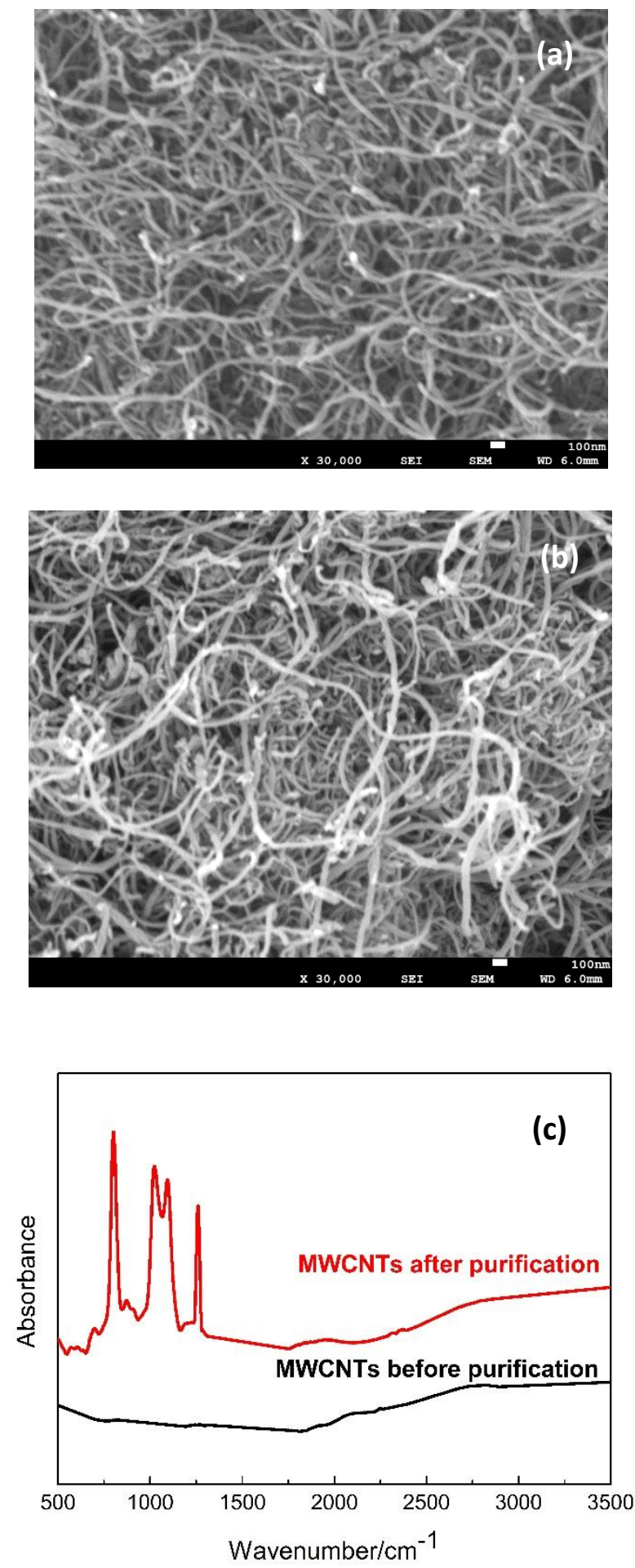

Fig. 4. Morphologies (a, b) and FT-IR spectra (c) of MWCNTs before and after purification 
Fig. 5

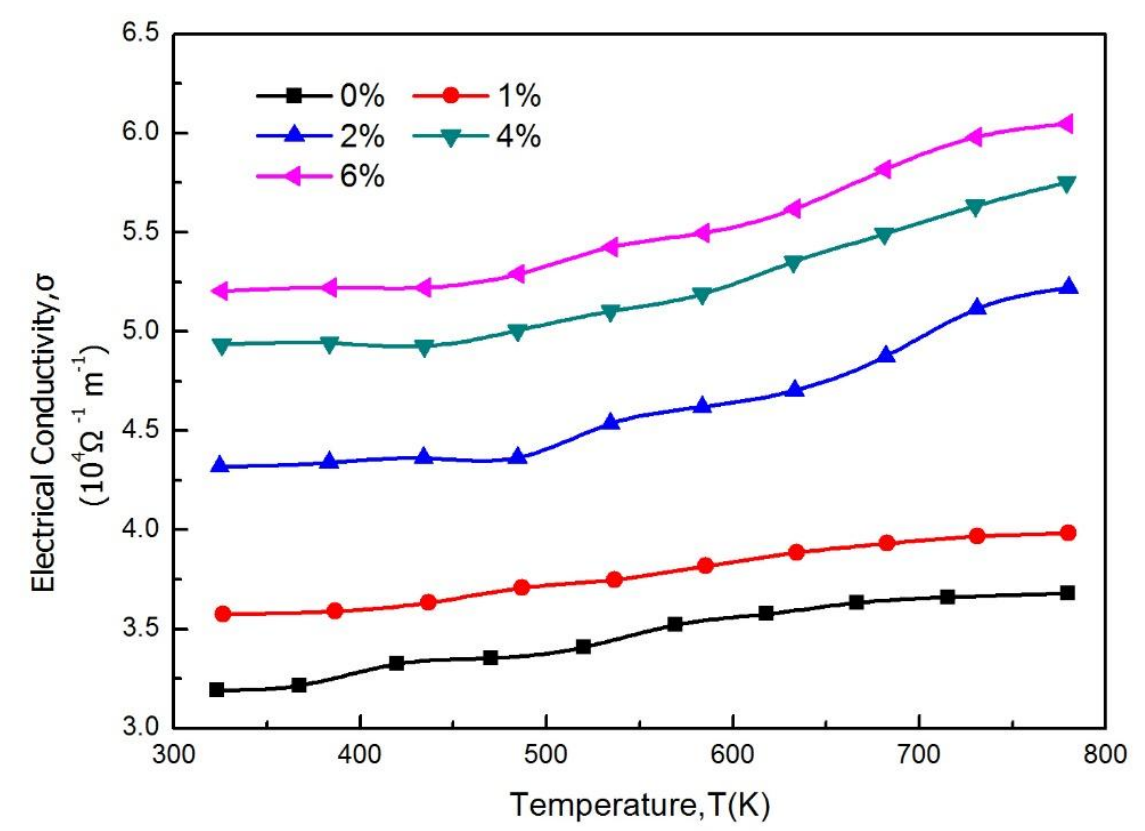

Fig. 5. Electrical conductivities of the samples containing $\mathrm{La}_{0.3} \mathrm{CoSb}_{3}$ and various weight percentage of MWCNTs compacted with SPS 
Fig. 6

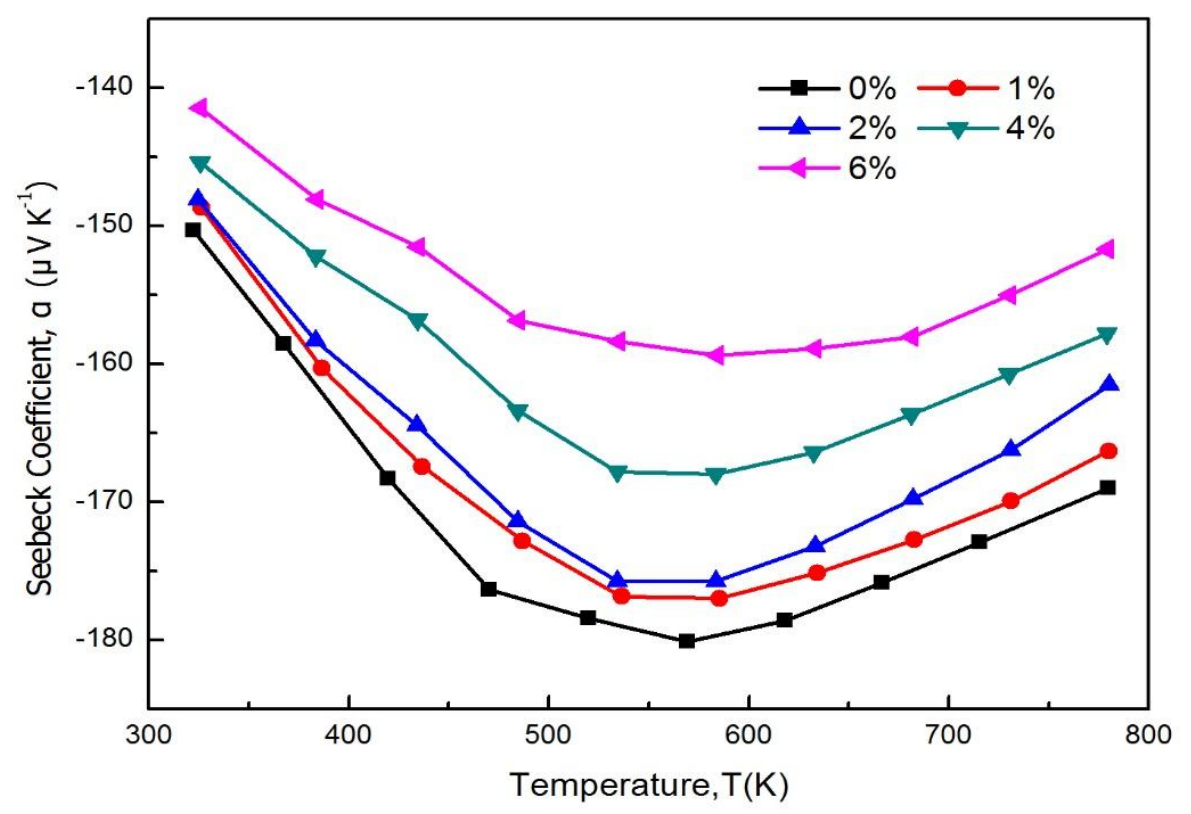

Fig. 6. Seebeck coefficients of the samples containing $\mathrm{La}_{0.3} \mathrm{CoSb}_{3}$ and various weight percentage of MWCNTs compacted with SPS 
Fig. 7

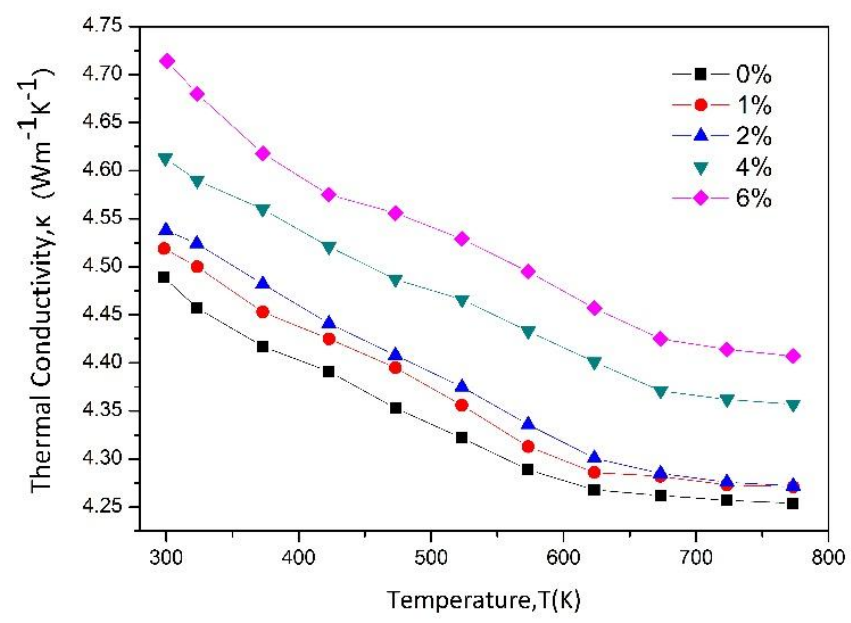

Fig. 7. Thermal conductivity $\mathrm{k}$ of the samples containing $\mathrm{La}_{0.3} \mathrm{CoSb}_{3}$ and various weight percentage of MWCNTs compacted with SPS 
Fig.8

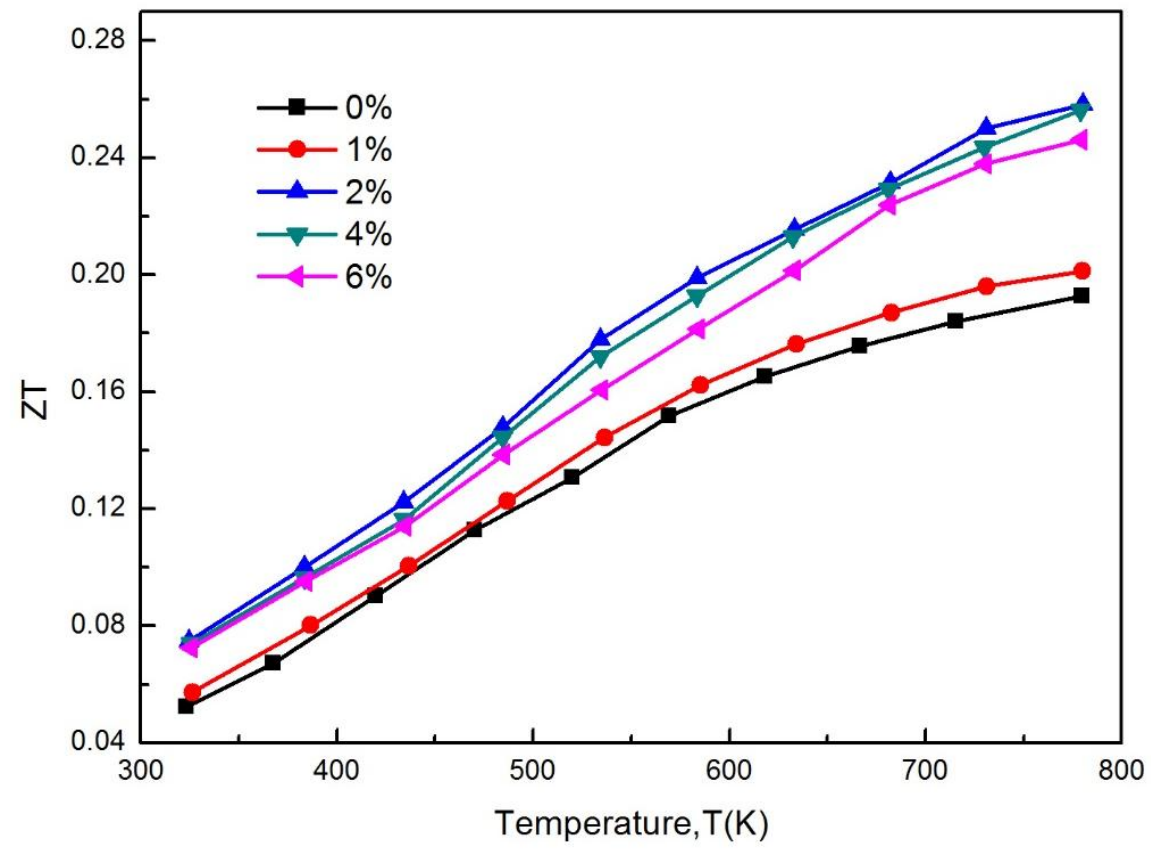

Fig. 8. ZT values of the samples containing $\mathrm{La}_{0.3} \mathrm{CoSb}_{3}$ and various weight percentage of MWCNTs compacted with SPS 